\title{
SUBDIRECT PRODUCTS OF RINGS AND DISTRIBUTIVE LATTICES
}

\author{
by HANS-J. BANDELT AND MARIO PETRICH*
}

(Received 20th October 1980)

\section{Introduction and summary}

Rings and distributive lattices can both be considered as semirings with commutative regular addition. Within this framework we can consider subdirect products of rings and distributive lattices. We may also require that the semirings with these restrictions are regarded as algebras with two binary operations and the unary operation of additive inversion (within the additive subgroup of the semiring). We can also consider distributive lattices with the two binary operations and the identity mapping as the unary operation. This makes it possible to speak of the join of ring varieties and distributive lattices. We restrict the ring varieties in order that their join with distributive lattices consist only of subdirect products. In certain cases these subdirect products can be obtained via a general construction of semirings by means of rings and distributive lattices.

In Section 2 we prove basic results concerning semirings which are lattices of rings. For a semiring of the type under study, i.e. with commutative regular addition, we define two congruences, one of which gives a quotient which is a distributive lattice and the other one gives a ring. The discussion is focused on these congruences and the additive structure mappings arising from the commutative regular addition.

Section 3 starts with a consideration of lattices of rings whose characteristics divide a fixed positive integer. This is followed by characterisations of lattices of rings belonging to a fixed variety of arithmetical rings. For semirings which are lattices of Boolean rings, an explicit construction and a categorical interpretation are provided.

A construction of semirings is given in Section 4 which amounts to a lattice of semirings where the operations are given as in the case of a strong semilattice of semigroups. The axioms governing this construction are very simple. It is then established that every semiring which is a lattice of rings with an identity has the structure prescribed by this construction. Examples are given illustrating the theory.

\section{Basic results}

A semiring is a system with associative addition and multiplication connected by distributivity of multiplication over addition.

\footnotetext{
*The second author gratefully acknowledges the support from a grant by the Deutsche Forschungsgemeinschaft.
} 
In this section $S$ stands for a semiring with commutative regular addition (these semirings have been considered by several authors, see V. N. Salii [8]). Hence the additive semigroup $S^{+}$of $S$ is a semilattice of groups. For each element $a$ of $S$, we denote by $-a$ its additive inverse in the additive group it belongs to. We will further adhere to the notation

$$
\bar{a}=a+(-a)
$$

The congruence $\rho$ on $S^{+}$which decomposes $S^{+}$into its maximal subgroups is the least semilattice congruence on $S^{+}$, and can be written succinctly as

$$
a \rho b \Leftrightarrow \bar{a}=\bar{b} .
$$

For any $a, b \in S$, we have

$$
\begin{gathered}
a b+(-a) b+a b=(a+(-a)+a) b=a b, \\
(-a) b+a b+(-a) b=((-a)+a+(-a)) b=(-a) b
\end{gathered}
$$

which by the uniqueness of negative gives the usual formula $(-a) b=-a b$; analogously $a(-b)=-a b$. Consequently

$$
\bar{a} b=\overline{a b}=a \bar{b} .
$$

It now follows that $\rho$ is also a multiplicative congruence on $S$.

The semiring $S$ may satisfy some of the following axioms.

$$
\begin{array}{ll}
a \bar{a}=\bar{a} & (a \in S), \\
a \bar{b}=\bar{b} a & (a, b \in S), \\
a+\bar{a} b=1 & (a, b \in S) .
\end{array}
$$

Lemma 1. The following statements hold.

(i) $S / \rho$ is idempotent if and only if $S$ satisfies (A1).

(ii) $S / \rho$ is commutative if and only if $S$ satisfies (A2).

(iii) $S / \rho$ is a lattice if and only if $S$ satisfies (A1), (A2), (A3).

Proof. We have seen above that for any $a, b \in S, \bar{a} b=a \bar{b}$ whence (i) and (ii) follow since $a \rho a^{2}$ can be written as $a \bar{a}=\bar{a}$ and $a b \rho b a$ can be written as $a \bar{b}=\bar{b} a$.

Assume next that $S / \rho$ is a lattice. Then (A1) and (A2) hold, and one of the absorption laws gives $a+a b \rho a$. Hence $\overline{a+a b}=\bar{a}$ so that

$$
a+a b+(-a)+(-a b)=a+(-a)
$$

Adding $a$ on both sides, we get $a+\bar{a} b=a$, i.e. (A3) holds. 
Conversely, assume that (A1), (A2), (A3) hold in S. By the above remarks and parts (i) and (ii), it remains to verify the absorption laws. The argument going in the opposite direction to the one above gives that (A3) yields $a+a b \rho a$. Now using the distributive law, we get the other absorption law. Hence $S / \rho$ is a lattice.

Note that the lattice in part (iii) above is distributive. The above lemma admits an interesting interpretation if we introduce the following terminology.

Definition. A semiring $S$ is a lattice $D$ of rings $R_{\alpha}$ if $S$ admits a congruence $\kappa$ such that $D=S / \kappa$ is a distributive lattice and each $\kappa$-class is a subring of $S$.

In an obvious way, we may replace "lattice" in the above definition by "semiring" (with certain properties). Since $\rho$ is the only congruence on $S$ all of whose classes are groups, we deduce from Lemma 1

Corollary. The following statements are valid.

(i) $S$ is a multiplicatively idempotent semiring of rings if and only if it satisfies (A1).

(ii) $S$ is a multiplicatively idempotent and commutative semiring of rings if and only if it satisfies (A1) and (A2).

(iii) $S$ is a lattice of rings if and only if it satisfies (A1), (A2) and (A3).

Note that a semiring $S$ is a union of pairwise disjoint rings whose zeros commute if and only if $S$ has commutative regular addition and satisfies (A1) and (A2). We can reduce the set of axioms for semirings with commutative regular addition and satisfying (A1), (A2) and (A3) by requiring: semirings with commutative addition and a unary operation "-" satisfying $-(-a)=a$ for all $a \in R$ or $\overline{-a}=\bar{a}$ for all $a \in R$ (where $\bar{a}=a+$ $(-a)$ as before).

The semirings we have arrived at in Lemma 1 part (iii) admit the following characterisation: $S$ is a semiring which admits a retraction $\phi$ onto an ideal $L$ such that $(\alpha)$ the classes of the congruence relation induced by $\phi$ are rings, and $(\beta) L$ is a lattice under the operations of $S$. Indeed, for the semirings in Lemma 1 part (iii), the mapping $\phi: a \rightarrow \bar{a}$ is a retraction onto the ideal and lattice $L$ of additive idempotents. Conversely, it suffices to observe that the addition of the semiring is automatically regular, and that it is commutative since $S^{+}$is then a semilattice of abelian groups.

We now introduce a second relation on $S$ as follows:

$$
a \sigma b \Leftrightarrow a+\bar{b}=\bar{a}+b .
$$

It is clear that $\sigma$ is reflexive and symmetric. For $a \sigma b$ and $c \in S$, we get

$$
\begin{aligned}
a c+\overline{b c} & =a c+\overline{b c}=(a+\bar{b}) c=(\bar{a}+b) c \\
& =\bar{a} c+b c=\overline{a c}+b c \\
(a+c)+\overline{b+c} & =a+c+b+c+(-b)+(-c) \\
& =a+\bar{b}+c=\overline{a+c}+b+c,
\end{aligned}
$$


whence we conclude that $\sigma$ is compatible with both operations. If $a \rho \cap \sigma b$, then $\bar{a}=\bar{b}$ and thus

$$
a=a+\bar{a}=a+\bar{b}=\bar{a}+b=\bar{b}+b=b
$$

so that $\rho \cap \sigma=\varepsilon$, the equality relation.

We will use the notation $x-y=x+(-y)$. The following characterisation of $\sigma$ will be useful.

Lemma 2. For any $a, b \in S$,

$$
a \sigma b \Leftrightarrow 2(a-b)=a-b
$$

Proof. If $a \sigma b$, then

$$
a+\bar{b}+(a-b)-b=\bar{a}+b+(a-b)-b
$$

which implies $2(a-b)=a-b$. Conversely, if $2(a-b)=a-b$, then $a-b=-(a-b)$ and thus

$$
\begin{aligned}
a+\bar{b} & =(a-b)+b=[(a-b)-(a-b)]+b \\
& =(\bar{a}+\bar{b})+b=\bar{a}+b
\end{aligned}
$$

so that $a \sigma b$.

We introduce another condition

$$
a+b=a \Rightarrow 2 b=b .
$$

This is equivalent to the condition: $a+b=a \Rightarrow b=b$.

Lemma 3. The relation $\sigma$ is transitive if and only if $S$ satisfies (A4).

Proof. Assume first that $\sigma$ is transitive and that $a+b=a$. Then $b+\bar{a}=\bar{a}=\bar{a}+\bar{b}$ so that $b \sigma \bar{a}$ and $\bar{a} \sigma \bar{b}$ which then gives $b \sigma \bar{b}$. But then $b=b+\bar{b}=2 \bar{b}=\bar{b}$ whence $2 b=b$.

Conversely, assume that $S$ satisfies (A4), and let $a \sigma b, b \sigma c$. Using Lemma 2, we obtain

$$
\begin{aligned}
(a-c)+[a-c+\bar{b}] & =(a-c)+\bar{b}+(a-c)+\bar{b} \\
& =(a-b)+(b-c)+(a-b)+(b-c) \\
& =(a-b)+(b-c)=a-c+\bar{b}
\end{aligned}
$$

which by (A4) gives $2(a-c)=a-c$ and thus $a \sigma c$ again by Lemma 2 . 
For any $a, b \in S$, we have

$$
\bar{a}+\bar{b}=\bar{a}+\bar{b}=\bar{a}+\bar{b}
$$

so that $\bar{a} \sigma \bar{b}$. This says that in the case that $\sigma$ is transitive, $S / \sigma$ has only one additive idempotent. Regularity of addition of $S$ implies the same for $S / \sigma$ and thus $S / \sigma$ is additively an abelian group. Since (A1)-(A4) are valid in any ring and any lattice, we have proved

Theorem 1. A semiring $S$ with regular addition is a subdirect product of a ring and $a$ lattice if and only if its addition is commutative and $S$ satisfies (A1)-(A4).

We have mentioned earlier that regularity and commutativity of addition of our semiring $S$ gives that $S^{+}$is a semilattice of groups. The structure of such semigroups is well-known, indeed, Clifford's theorem says that the operation is given by a system of homomorphisms $\phi_{\alpha, \beta}$ satisfying certain conditions. Here $D=S / \rho$ is an upper semilattice, we denote the $\rho$-classes by $R_{\alpha}$, so that $\phi_{\alpha, \beta}: R_{\alpha} \rightarrow R_{\beta}(\alpha \leqq \beta)$ is given by

$$
\phi_{\alpha, \beta}: r \rightarrow r+0_{\beta} \quad\left(r \in R_{\alpha}\right),
$$

where $0_{\beta}$ denotes the (additive) identity of $R_{\beta}^{+}$. It is readily verified that all functions $\phi_{\alpha, \beta}$ are one-to-one if and only if $S$ satisfies (A4). The next lemma shows that these functions also enjoy some multiplicative properties.

Let $\phi$ be a homomorphism of a ring $R$ into a ring $R^{\prime}$. We let im $\phi$ denote the image of $R$ in $R^{\prime}$ under $\phi$ and $\operatorname{ker} \phi$ be the kernel of $\phi$. We also write

$$
\mathscr{A}(R)=\{r \in R \mid r R=R r=0\}
$$

for the (double) annihilator of $R$.

Lemma 4. If $S$ satisfies (A1), (A2) and (A3), then the following statements are valid for any $\alpha \leqq \beta$.

(i) $\phi_{\alpha, \beta}$ is a ring homomorphism.

(ii) $\operatorname{im} \phi_{\alpha, \beta}$ is an ideal of $R_{\beta}$.

(iii) $\operatorname{ker} \phi_{\alpha, \beta} \subseteq \mathscr{A}\left(R_{\alpha}\right)$.

Proof. First recall that (A1) ensures that each $R_{\alpha}$ is closed under multiplication, and is thus a ring.

(i) We have seen above that $\phi=\phi_{\alpha, \beta}$ is additive. Let $a, b \in R_{\alpha}$. Then $a 0_{\beta} \in R_{\alpha}$ and thus

$$
a 0_{\beta}=a \overline{0_{\beta}}=\overline{a 0_{\beta}}=0_{\alpha} \text {. }
$$


Similarly $0_{\beta} b=0_{\alpha}$, so that

$$
\begin{aligned}
\left(a \phi_{\alpha, \beta}\right)\left(b \phi_{\alpha, \beta}\right) & =\left(a+0_{\beta}\right)\left(b+0_{\beta}\right) \\
& =a b+0_{\beta} b+a 0_{\beta}+0_{\beta} 0_{\beta} \\
& =a b+0_{\beta}=(a b) \phi_{\alpha, \beta} .
\end{aligned}
$$

Consequently $\phi_{\alpha, \beta}$ is a ring homomorphism.

(ii) If now $a \in R_{\alpha}$ and $b \in R_{\beta}$, then $b\left(a+0_{\beta}\right)=b a+0_{\beta}$ where $b a \in R_{\alpha}$ so that $b\left(a \phi_{\alpha, \beta}\right)$ $=(b a) \phi_{\alpha, \beta}$. We analogously obtain that $\left(a \phi_{\alpha, \beta}\right) b=(a b) \phi_{\alpha, \beta}$. Since im $\phi_{\alpha, \beta}$ is already a subring of $R_{\beta}$, we deduce that it is an ideal of $R_{\beta}$.

(iii) Let $a \in \operatorname{ker} \phi_{\alpha, \beta}$ and $b \in R_{\alpha}$. Then $a+0_{\beta}=0_{\beta}$ and thus $b\left(a+0_{\beta}\right)=b 0_{\beta}$ which implies $b a+0_{\alpha}=0_{\alpha}$ so that $b a=0_{\alpha}$. Analogously $a b=0_{\alpha}$, which proves that $a \in \mathscr{A}\left(R_{\alpha}\right)$, and thus $\operatorname{ker} \phi_{\alpha, \beta} \subseteq \mathscr{A}\left(R_{\alpha}\right)$.

Corollary 1. If $S$ is a lattice of rings with trivial annihilator, then $S$ is a subdirect product of a ring with trivial annihilator and a lattice.

Proof. By part (iii) of Lemma 4, all the functions $\phi_{\alpha, \beta}$ are one-to-one. We have remarked above that this is equivalent to condition (A4). Now by Theorem 1 we have that $S$ is a subdirect product of a ring $R$ and lattice $D$. Assume that $S \subseteq R \times D$ and take $r \in \mathscr{A}(R)$. Then for some $\alpha \in D$, we have $(r, \alpha) \in S$. Then for all elements of $S$ of the form $(a, \alpha)$, we get $(r, \alpha)(a, \alpha)=(a, \alpha)(r, \alpha)=(0, \alpha)$ since $r \in \mathscr{A}(R)$. But $R_{\alpha}=(R \times\{\alpha\}) \cap S$ is one of the rings of which $S$ is a lattice of. Hence $(r, \alpha) \in \mathscr{A}\left(R_{\alpha}\right)=(0, \alpha)$ by the hypothesis, so that $r=0$. Consequently $\mathscr{A}(R)=0$.

Recall that a ring is called simple if it has no proper ideals and $R^{2} \neq 0$.

Corollary 2. If $S$ is a lattice of simple rings, then $S$ is the direct product of a simple ring and a lattice.

Proof. First note that the annihilator of a simple ring $R$ must be trivial and that $R \neq 0$. Thus Corollary 1 implies that $S$ is a subdirect product of a ring $R$ and a lattice $D$. But part (ii) of Lemma 4 yields that for any $\alpha \leqq \beta, \phi_{\alpha, \beta}$ maps $R_{\alpha}$ onto $R_{\beta}$, and is thus a ring isomorphism. This together with the fact that $S$ is a subdirect product of $R$ and $D$ gives the assertion that $S$ is the direct product of $R$ and $D$.

Example 1. A lattice of rings is in general not a subdirect product of a ring and a (distributive) lattice. For, consider the commutative semiring $S=\{0, a, b\}$ with zero 0 for which $a^{2}=2 a=a b=0$ and $b^{2}=2 b=a+b=b$ (see Figure 1). Then $S$ is a lattice of null rings $R_{\alpha}=\{0, a\}$ and $R_{\beta}=\{b\}(\alpha<\beta)$, though $S$ is subdirectly irreducible. This seems to contradict the first assertion of $([6]$, Theorem 6$)$. 


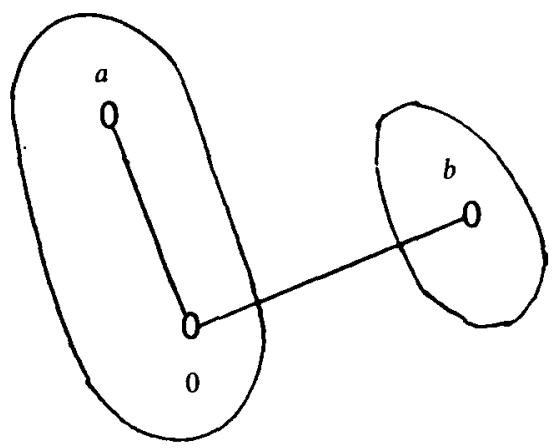

Figure 1

\section{The join of distributive lattices-and arithmetical ring varieties}

We consider here semirings which are lattices of rings belonging to some particular varieties of rings. The first such class is that of rings whose characteristics divide a fixed positive integer. The second class is an arbitrary variety of arithmetical rings. The third is the variety of Boolean rings where we can give an explicit construction of semirings under consideration.

For a ring of finite characteristic, the additive inverse can be expressed by means of addition. Since in this section we consider only such rings, we regard the rings, the distributive lattices and in general the semirings as algebras with just two binary operations, of which the addition is commutative.

We start by introducing three more axioms as follows: for an integer $n>1$, let

$$
\begin{gathered}
n a^{2}=n a, \\
n a b=n b a, \\
a+n a b=a .
\end{gathered}
$$

Proposition 1. Let $n>1$ and $S$ be a semiring with commutative addition. Then $S$ is $a$ lattice of rings whose characteristics divide $n$ if and only if $S$ satisfies (A5), (A6), (A7).

Proof. For the direct part, we note that in a ring $R$ whose characteristic divides $n$, we have $\bar{a}=n a$. Axioms (A5), (A6), (A7) thus coincide with (A1), (A2), (A3). Conversely, assume the validity of (A5), (A6), (A7). Letting $b=a$ in (A7) and taking into account (A5), we get $(n+1) a=a$ which yields regularity of the addition and $\bar{a}=n a$. Again axioms (A5), (A6), (A7) coincide with axioms (A1), (A2), (A3) which by the corollary to Lemma 1 gives that $S$ is a lattice of rings. These rings satisfy the identity $n a=0$, hence their characteristics divide $n$.

H. Werner and R. Wille [7] have shown that a ring variety $\mathscr{V}$ is generated by finitely many finite fields if and only if $\mathscr{V}$ consists of all rings satisfying the identities

$$
\sum_{p \in P}\left(\prod_{q \in P} q\{)^{\left(p^{k(p)}-1\right)} \cdot x^{p^{k(p)}}=x\right.
$$

EMS-D 
where

$$
\begin{gathered}
k(p)=\prod_{k \in N(p)} k, \\
x \cdot \prod_{p \in P} \prod_{k \in N(p)}\left(x^{p^{k}}-x\right)=0
\end{gathered}
$$

for some finite set $P$ of prime numbers and finite sets $N(p)(p \in P)$ of positive integers.

The next proposition lists some further characterisations of these varieties, which are essentially due to G. M. Bergman, G. Michler, H. Werner and R. Wille. We give a proof for those implications which are not proved in [3], [7].

Proposition 2. For a ring variety $\mathscr{V}$ the following statements are equivalent.

(i) Every ring in $\mathscr{V}$ has a trivial annihilator.

(ii) $\mathscr{V}$ contains no nontrivial null rings.

(iii) No ring in $\mathscr{V}$ has nonzero nilpotent elements.

(iv) There exists a polynomial $t(x)$ without the linear term such that the identity $t(x)=x$ is valid in $\mathscr{V}$.

(v) $\mathscr{V}$ is arithmetical, that is, the ideal lattice of any ring in $\mathscr{V}$ is distributive.

(vi) $\mathscr{V}$ is the variety of all rings satisfying (W1) and (W2) for some $P$ and $N(p)(p \in P)$.

Proof. The equivalence of (iv), (v), (vi) is proved in [3], [7]. The implications (iii) $\Rightarrow$ (i) $\Rightarrow$ (ii) and (iv) $\Rightarrow$ (ii) are obvious. If $x^{n}=0$ and $x^{n-1} \neq 0(n>1)$ in a ring $R$, then the subring generated by $x^{n-1}$ is a null ring. Hence (ii) implies (iii). It remains to verify (ii) $\Rightarrow$ (iv). Let $\mathscr{V}$ be given by the system $\left\{u_{i}\left(x_{i, 1}, \ldots, x_{i, n_{i}}\right)=0\right\}_{i \in I}$ of identities. Let $k_{i, j} x_{i, j}$ be the linear terms occurring in $u_{i}$. Let $m$ be the greatest common divisor of all $k_{i, j}(i \in I$, $\left.j=1, \ldots, n_{i}\right)$. Obviously, the abelian group $\mathbb{Z} / m \mathbb{Z}$ considered as a null ring belongs to $\mathscr{V}$. Thus by (ii) $m=1$ must be true. Hence for some finite subset $I_{0}$ of $I$ and integers $q_{i, j}$ we get

$$
\sum_{i \in I_{0}} \sum_{j=1}^{n_{i}} k_{i, j} \cdot q_{i, j}=1
$$

Then by substituting $-q_{i, j} x$ for $x_{i, j}$ and adding up the identities $u_{i}=0$ for $i \in I_{0}$, we get an identity $t(x)-x=0$ where $t(x)$ is without linear term.

Lemma 5. Let $S$ be a semiring with commutative addition. If $S$ satisfies (A7) and an identity of the form (W1), then $S$ satisfies (A5).

Proof. Let $a \in S$. According to (W1), $a$ is equal to a polynomial in the variable $a$ with no linear term. Hence $a=a u$, where $u$ is a polynomial in $a$, and thus $u=a v+k a$ for some polynomial $v$ in $a$ and integer $k \geqq 0$. Now by (A7), we get

$$
(n+1) a=n a+a=a+n a u=a,
$$


whence, again by (A7)

$$
\begin{aligned}
a^{2} & =(k n+1) a^{2}=k n a^{2}+a^{2}=k n a^{2}+\left(n a^{2} v+a^{2}\right) \\
& =n a(a v+k a)+a^{2}=n a u+a^{2}=n a+a^{2} .
\end{aligned}
$$

By (2) and (1), we obtain

$$
n a^{2}=n^{2} a+n a^{2}=n a+n a^{2} .
$$

On the other hand, again using (A7) and (1)

$$
n a=n a+n^{2} a^{2}=n a+n a^{2},
$$

which together with (3) yields $n a^{2}=n a$, that is (A5) holds.

Let $\mathscr{D}$ denote the variety of distributive lattices.

Theorem 2. Let $\mathscr{V}$ be an arithmetical variety given by the identities (W1) and (W2). The following statements concerning a semiring $S$ with commutative addition are equivalent.

(i) $S$ is a lattice of rings in $\mathscr{V}$.

(ii) $S$ is a subdirect product of a ring in $\mathscr{V}$ and a (distributive) lattice.

(iii) $S$ is in the join $\mathscr{V} \vee \mathscr{D}$ of the semiring varieties $\mathscr{V}$ and $\mathscr{D}$.

(iv) $S$ satisfies (A6), (A7), (W1), and

$$
x \cdot \prod_{p \in P} \prod_{k \in N(p)}\left(x^{p^{k}}+(n-1) x\right)=n x, \text { where } n=\prod_{p \in P} p
$$

Proof. (i) implies (ii). By Corollary 1 to Lemma $4, S$ is a subdirect product of a ring $R$ and a lattice $D$. Since $D$ is a homomorphic image of $S$, it must be distributive. Both the identities (W1) and (W2) are in a single variable, whence it follows easily that $R$ satisfies (W1), (W2).

(ii) implies (iii) trivially.

(iii) implies (iv). Since both the rings in $\mathscr{V}$ and the lattices (in $\mathscr{D}$ ) satisfy (A6), (A7), (W1), (W2'), so do all semirings in their join $\mathscr{V} \vee \mathscr{D}$.

(iv) implies (i). By Lemma 5 and Proposition $1, S$ is a lattice of rings. These rings must satisfy (W1), $(n+1) a=a$ and (W2'), and therefore must be in $\mathscr{V}$.

For any integers $m, n>1$, the variety $\mathscr{R}_{m . n}$ of all rings satisfying $x^{m}=x$ and $n x=0$ is arithmetical. A semiring is in the join $\mathscr{R}_{m, n} \vee \mathscr{D}$ if and only if it satisfies the identities (A6), (A7) and $x^{m}=x$. Unfortunately, not every arithmetical ring variety occurs among the $\mathscr{R}_{m, n}$. For instance, $\left\{G F(3), G F\left(7^{2}\right)\right\} \subseteq \mathscr{R}_{m, n}$ if and only if $\left\{G F\left(3^{2}\right), G F\left(7^{2}\right)\right\} \subseteq \mathscr{R}_{m, n}$. 
An important case of the preceding class of semirings is provided by lattices of Boolean rings $(m=n=2)$. This gives a common abstraction of Boolean rings and distributive lattices; for Boolean rings with identity and Boolean lattices, see the classical result of M. H. A. Newman [4].

Rccall that every relatively complemented distributive lattice $(L, \vee, \cdot)$ with zero can bc made into a Boolean ring $(L,+, \cdot)$, where $a+b$ is the relative complement of $a b$ in the interval $[0, a \vee b]$.

Theorem 3. Let $(L, \vee, \cdot)$ be a distributive lattice and let $\phi$ be a retraction of $L$ upon an ideal $D$ such that every class of the congruence $\tau$ induced by $\phi$ is relatively complemented. For any two elements $a, b$ of a class $C$ of $\tau$, let $a \oplus b$ denote the sum of $a$ and $b$ in the Boolean ring $C$. On $L$ define a new operation + by

$$
a+b=(a \vee b \phi) \oplus(a \phi \vee b) \quad(a, b \in L)
$$

Then $(L,+, \cdot)$ is a semiring which is a lattice of Boolean rings, and conversely, every such semiring can be so obtained.

Proof. Let $(L, \vee, \cdot)$ be a distributive lattice and let + be given by (4). Since

$$
(a \vee b \phi) \phi=a \phi \vee b \phi=(a \phi \vee b) \phi,
$$

the right hand side of (4) is unambiguously defined. If $a \phi=b \phi$ and $c \phi=c$, then

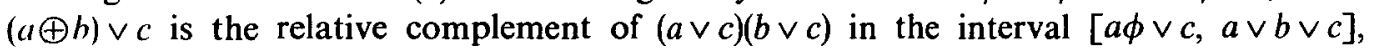
and so $(a \oplus b) \vee c=(a \vee c) \oplus(b \vee c)$ (see Figure 2).

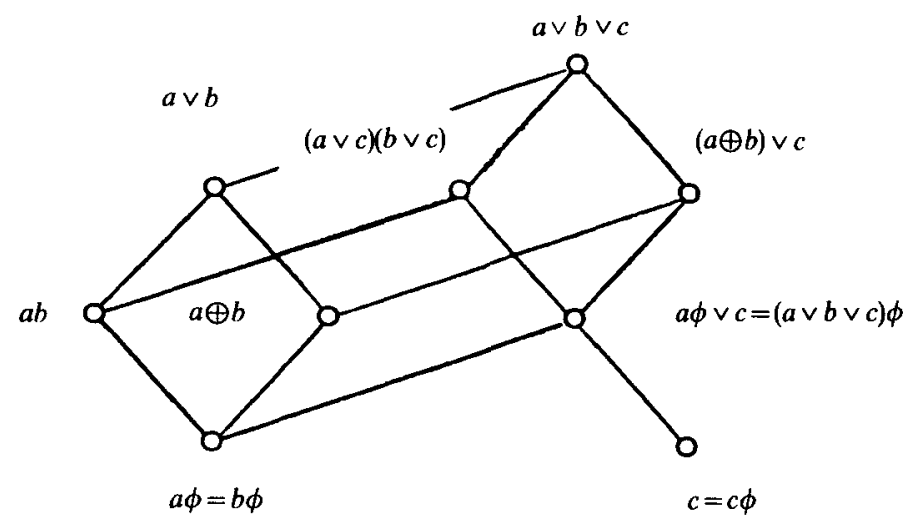

Figure 2

Hence for arbitrary $a, b, c \in L$, we have

$$
\begin{aligned}
(a+b)+c & =((a \vee b \phi) \oplus(a \phi \vee b))+c \\
& =((a \vee b \phi) \phi \vee c) \oplus[((a \vee b \phi) \oplus(a \phi \vee b)) \vee c \phi] \\
& =(a \phi \vee b \phi \vee c) \oplus(a \vee b \phi \vee c \phi) \oplus(a \phi \vee b \vee c \phi)
\end{aligned}
$$


The same expression can also be obtained for $a+(b+c)$, and thus + is associative. If $a \phi$ $=b \phi$, then for any $c \in L,(a \oplus b) c$ is the relative complement of $a b c$ in the interval [a $a c$, $a c \vee b c]$, and so $(a \oplus b) c=a c \oplus b c$ (see Figure 3).

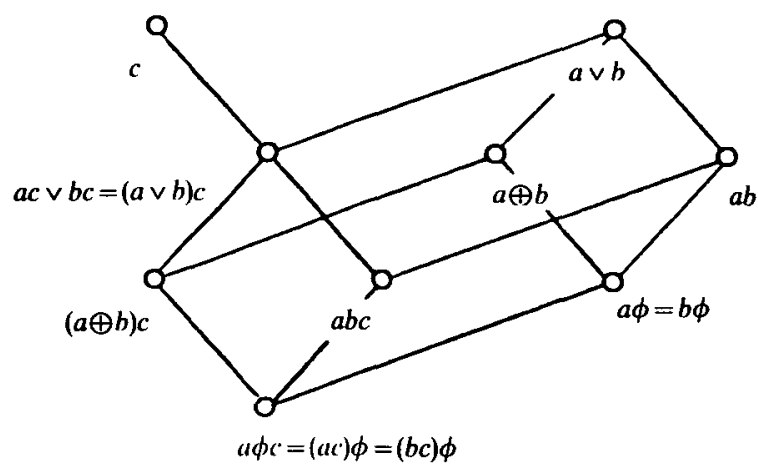

Figure 3

Hence for arbitrary $a, b, c \in L$, we have

$$
\begin{aligned}
(a+b) c & =((a \vee b \phi) \oplus(a \phi \vee b)) c=(a \vee b \phi) c \oplus(a \phi \vee b) c \\
& =(a c \vee(b c) \phi) \oplus((a c) \phi \vee b c)=a c+b c .
\end{aligned}
$$

We conclude that $(L,+, \cdot)$ is a semiring. If $a \phi=b \phi$, then

$$
a+b=(a \vee a \phi) \oplus(b \vee b \phi)=a \oplus b,
$$

so that + extends $\oplus$. Since for any $a, b \in L$,

$$
(a+b) \phi=a \phi \vee b \phi=a \phi+b \phi,
$$

$\phi$ is a retraction of the semiring $(L,+, \cdot)$ upon $D$. Every class of the induced congruence is a Boolean ring, and therefore $(L,+, \cdot)$ is a lattice of Boolean rings.

Conversely, let $L$ be a semiring which is a lattice of Boolean rings. It is routine to check that $(L, \vee, \cdot)$ is a distributive lattice, where

$$
x \vee y=x+y+x y .
$$

Then $D=\{x \in L \mid x=2 x\}$ is an ideal of $(L, \vee$,$) and \phi: x \rightarrow 2 x$ gives the retraction of $\left(L, \vee,{ }^{\circ}\right)$ upon $D$. Every class of the congruence induced by $\phi$ is a Boolean ring with respect to + and , and we have

$$
\begin{aligned}
x+y & =(x+2 x y)+(y+2 x y)=x+2 y+2 x y+2 x+y+2 x y \\
& =(x \vee 2 y)+(2 x \vee y)=(x \vee y \phi)+(x \phi \vee y) .
\end{aligned}
$$


Let $\mathscr{L}$ be the category of the following universal algebras $(L, \vee, \wedge,-)$ of type $(2,2,1)$ and their homomorphisms:

(i) $(L, \vee, \wedge)$ is a distributive lattice,

(ii) $x \rightarrow \bar{x}$ is a translation of $(L, \wedge)$ (that is $\bar{a} \wedge b=\overline{a \wedge b}$, or equivalently, $x \rightarrow \bar{x}$ is a retraction upon an ideal of $(L, \vee, \wedge))$,

(iii) for each $a \in L$, the sublattice $\{x \in L \mid \bar{a}=\bar{x}\}$ of $L$ is relatively complemented.

Further let $\mathscr{S}$ be the category of semirings $(S,+, \cdot)$ which are lattices of Boolean rings and semiring homomorphisms.

Lemma 6. Let $L_{1}$ and $L_{2}$ be $\mathscr{L}$-objects. Under the correspondence of Theorem 3, a map $\theta: L_{1} \rightarrow L_{2}$ is an $\mathscr{L}$-morphism if and only if $\theta$ is an $\mathscr{S}$-morphism.

Proof. If $\theta$ is an $\mathscr{L}$-morphism, then $\theta$ is a lattice homomorphism and $\bar{a}=\bar{b}$ implies $\bar{a} \bar{\theta}=\overline{b \theta}$ for all $a, b \in L_{1}$. Therefore $\theta$ preserves the Boolean addition $\oplus$ of ring components, and consequently,

$$
(a+b) \theta=(\bar{a} \vee b) \theta \oplus(a \vee \bar{b}) \theta=a \theta+b \theta .
$$

Conversely, if $\theta$ is an $\mathscr{P}$-morphism, then $\theta$ is an $\mathscr{L}$-morphism since $a \vee b=a+b+a b$, $a \wedge b=a b$, and $\bar{a}=a+a$.

From Theorem 3 and Lemma 6 we conclude:

Corollary. $\mathscr{L}$ and $\mathscr{S}$ are isomorphic categories.

\section{A construction}

The now classical theorem of A. H. Clifford which determines the structure of semigroups which are semilattices of groups can be carried over to suitable classes of semirings. This was done by McAlister [2] for a distributive lattice of lattice ordered groups. We offer below a variant of this construction for a distributive lattice of (semi) rings suitable for proving a converse for rings with identity.

Construction. Let $D$ be a distributive lattice and let $S_{\alpha}$ be a family of pairwise disjoint semirings indexed by elements in $D$. For each pair $x \leqq \beta$ in $D$, let

$$
\phi_{\alpha, \beta}: S_{\alpha} \rightarrow S_{\beta}, \quad \psi_{\beta, \alpha}: S_{\beta} \rightarrow S_{\alpha}
$$

be (semiring) homomorphisms satisfying the following requirements:

$$
\begin{gathered}
\phi_{\alpha, \alpha}=\psi_{\alpha, \alpha}=i_{S_{\alpha}}, \text { the identity function on } S_{\alpha,} \\
\phi_{\alpha, \beta} \phi_{\beta, \gamma}=\phi_{\alpha, \gamma}, \psi_{\gamma, \beta} \psi_{\beta, \alpha}=\psi_{\gamma, \alpha} \text { if } \alpha<\beta<\gamma, \\
S_{\alpha} \phi_{\alpha, \beta} \text { is an ideal of } S_{\beta} \text { if } \alpha<\beta, \\
\phi_{\alpha, \gamma} \psi_{\gamma, \beta}=\psi_{\alpha, \alpha \beta} \phi_{\alpha \beta, \beta} \text { if } \alpha+\beta \leqq \gamma .
\end{gathered}
$$


On $S=\left(\int_{x, 1)} S_{x}\right.$ define the operations: for $a \in S_{\alpha}, b \in S_{\beta}$,

$$
\begin{gathered}
a+b=a \phi_{\alpha, \alpha+\beta}+b \phi_{\beta, \alpha+\beta}, \\
a \cdot b=a \psi_{\alpha, \alpha \beta} \cdot b \psi_{\beta, \alpha \beta} .
\end{gathered}
$$

Denote this system by $S=\left\langle D ; S_{\alpha}, \phi_{\alpha, \beta}, \psi_{\beta, \alpha}\right\rangle$.

Note that the construction discussed in $[1]$ is more general than the one above.

Theorem 4. With the above notation, $S$ is a semiring. Moreover, the relation $\sigma$ defined on $S$ by

$$
a \sigma b \Leftrightarrow a \phi_{\alpha, \alpha+\beta}=b \phi_{\beta, \alpha+\beta} \quad\left(a \in S_{\alpha}, b \in S_{\beta}\right)
$$

is a congruence, and $S$ is a subdirect product of $D$ and $S / \sigma$.

Proof. It can be checked readily, and is a well-known fact from semigroup theory, that the above defined addition and multiplication are associative and the former is evidently commutative.

As a preparation for the proof of distributivity, we first observe that for $\alpha=\beta$ in (R4), we get

$$
\phi_{\alpha, \alpha}=\psi_{\alpha, a}=t_{s_{\alpha}} \text { if } \quad \alpha<\beta .
$$

Now for $\alpha<\beta$ and $a \in S_{\alpha}, b \in S_{\beta}$, we get by (R3) that $b \cdot a \phi_{\alpha, \beta}=a^{\prime} \phi_{\alpha, \beta}$ for some $a^{\prime} \in S_{\alpha}$. Hence by (1), we obtain

$$
\begin{aligned}
a^{\prime} & =a^{\prime} \phi_{\alpha, \beta} \psi_{\beta, \alpha}=\left(b \cdot a \phi_{\alpha, \beta}\right) \psi_{\beta, \alpha} \\
& =b \psi_{\beta, \alpha} \cdot a \phi_{\alpha, \beta} \psi_{\beta, \alpha}=b \psi_{\beta, \alpha} \cdot a
\end{aligned}
$$

so that

$$
b \cdot a \phi_{\alpha, \beta}=\left(b \psi_{\beta, \alpha} \cdot a\right) \phi_{\alpha, \beta} \text { if } \quad \alpha<\beta
$$

We will now prove the distributive law $a \cdot(b+c)=a \cdot b+a \cdot c$ where $a \in S_{\alpha}, b \in S_{\beta}, c \in S_{\gamma}$. We compute

$$
\begin{aligned}
(a \cdot b) \phi_{\alpha \beta, \alpha(\beta+\gamma)} & =\left(a \psi_{\alpha, \alpha \beta} \cdot b \psi_{\beta, \alpha \beta}\right) \phi_{\alpha \beta, \alpha(\beta+\gamma)} \\
& =\left(a \psi_{\alpha, \alpha(\beta+\gamma)} \psi_{\alpha(\beta+\gamma), \alpha \beta} \cdot b \psi_{\beta, \alpha \beta}\right) \phi_{\alpha \beta, \alpha(\beta+\gamma)} \\
& =a \psi_{\alpha, \alpha(\beta+\gamma)} \cdot b \psi_{\beta, \alpha \beta} \phi_{\alpha \beta, \alpha(\beta+\gamma)} \\
& =a \psi_{\alpha, \alpha(\beta+\gamma)} \cdot b \phi_{\beta, \beta+\gamma} \psi_{\beta+\gamma, \alpha(\beta+\gamma)}
\end{aligned}
$$


and similarly

$$
(a \cdot c) \phi_{\alpha \gamma, \alpha(\beta+\gamma)}=a \psi_{\alpha, \alpha(\beta+\gamma)} \cdot c \phi_{\gamma, \beta+\gamma} \psi_{\beta+\gamma, \alpha(\beta+\gamma)}
$$

Using this, we obtain

$$
\begin{aligned}
a \cdot(b+c)= & a \cdot\left(b \phi_{\beta, \beta+\gamma}+c \phi_{\gamma, \beta+\gamma}\right) \\
= & a \psi_{\alpha, \alpha(\beta+\gamma)} \cdot\left(b \phi_{\beta, \beta+\gamma}+c \phi_{\gamma, \beta+\gamma}\right) \psi_{\beta+\gamma, \alpha(\beta+\gamma)} \\
= & a \psi_{\alpha, \alpha(\beta+\gamma)} \cdot\left(b \phi_{\beta, \beta+\gamma} \psi_{\beta+\gamma, \alpha(\beta+\gamma)}+c \phi_{\gamma, \beta+\gamma} \psi_{\beta+\gamma, \alpha(\beta+\gamma)}\right) \\
= & a \psi_{\alpha, \alpha(\beta+\gamma)} \cdot b \phi_{\beta, \beta+\gamma} \psi_{\beta+\gamma, \alpha(\beta+\gamma)}+a \psi_{\alpha, \alpha(\beta+\gamma)} \\
& \cdot c \phi_{\gamma, \beta+\gamma} \psi_{\beta+\gamma, \alpha(\beta+\gamma)} \\
= & (a \cdot b) \phi_{\alpha \beta, \alpha(\beta+\gamma)}+(a \cdot c) \phi_{\alpha \gamma, \alpha(\beta+\gamma)} \\
= & a \cdot b+a \cdot c .
\end{aligned}
$$

An analogous argument can be used to prove the other distributive law. We conclude that $S$ is a semiring.

Next let $a \in S_{\alpha}, b \in S_{\beta}, c \in S_{\gamma}$ and assume that $a \sigma b$. Then

$$
\begin{aligned}
(a \cdot c) \phi_{\alpha \gamma,(\alpha+\beta) \gamma} & =\left(a \psi_{\alpha, \alpha \gamma} \cdot c \psi_{\gamma, \alpha \gamma}\right) \phi_{\alpha \gamma,(\alpha+\beta) \gamma} \\
& =a \psi_{\alpha, \alpha \gamma} \phi_{\alpha \gamma,(\alpha+\beta) \gamma} \cdot c \psi_{\gamma,(\alpha+\beta) \gamma} \\
& =a \phi_{\alpha, \alpha+\beta} \psi_{\alpha+\beta,(\alpha+\beta) \gamma} \cdot c \psi_{\gamma,(\alpha+\beta) \gamma} \\
& =b \phi_{\beta, \alpha+\beta} \psi_{\alpha+\beta,(\alpha+\beta) \gamma} \cdot c \psi_{\gamma,(\alpha+\beta) \gamma} \\
& =\cdots=(b \cdot c) \phi_{\beta \gamma,(\alpha+\beta) \gamma}
\end{aligned}
$$

and similarly

$$
(c \cdot a) \phi_{\alpha \gamma,(\alpha+\beta) \gamma}=(c \cdot b) \phi_{\beta \gamma,(\alpha+\beta) \gamma} .
$$

Now all $\phi_{\alpha, \beta}$ with $\alpha<\beta$ are one-to-one by (1). Hence by ([5] III.7.11), we deduce that $\sigma$ is a congruence and that $S$ is a subdirect product of $D$ and $S / \sigma$.

We have seen in Corollary 1 to Lemma 4 that a semiring which is a lattice of rings with trivial annihilator is actually a subdirect product of such a ring and a (distributive) lattice. In Corollary 2 to the same lemma, the conclusion is even stronger, viz. a lattice of simple rings is the direct product of a simple ring and a (distributive) lattice. For lattices of rings having an identity, we have an intermediate case, as we now show. 
Theorem 5. Let $S$ be a semiring which is a lattice of rings with identity. Then $S$ can be obtained as in the construction above.

Proof. Let $S$ be a lattice $D$ of rings $R_{\alpha}$ with identity $1_{\alpha}$. For $\alpha \leqq \beta$, the function $\psi_{\beta, \alpha}$ defined on $S_{\beta}$ by

$$
\psi_{\beta, \alpha}: b \rightarrow 1_{a} b
$$

is a multiplicative homomorphism (see, e.g. [5], III.4.5) of $R_{\beta}$ into $R_{\alpha}$. Clearly $\psi_{\beta, \alpha}$ is also additive, and is thus a ring homomorphism. Since a ring with identity has trivial annihilator, we have by Lemma 4 that all $\phi_{\alpha, \beta}$ are one-to-one.

Axiom (R1) is obviously satisfied. For $\alpha<\beta$ and $a \in R_{\alpha}$, we have

$$
\left(1_{\beta} a\right) \phi_{\alpha, \beta}=1_{\beta} a+0_{\beta}=1_{\beta}\left(a+0_{\beta}\right)=a+0_{\beta}=a \phi_{\alpha, \beta}
$$

which then implies that $1_{\beta} a=a$. If now $\alpha<\beta<\gamma$ and $c \in R_{\gamma}$, we get

$$
c \psi_{\gamma, \beta} \psi_{\beta, \alpha}=i_{\alpha}\left(1_{\beta} c\right)=1_{\alpha} c=c \psi_{\gamma, \alpha}
$$

which gives the second half of (R2); the first half is automatically satisfied. Validity of (R3) follows from Lemma 4. For any $\alpha+\beta \leqq \gamma$ and $a \in R_{\alpha}$, we compute (using $1_{\beta} a=a$ if $\alpha<\beta)$,

$$
\begin{aligned}
a \phi_{\alpha, \gamma} \psi_{\gamma, \beta} & =1_{\beta}\left(a+0_{\gamma}\right)=1_{\beta} a+1_{\beta} 0_{\gamma}=1_{\beta} a+0_{\beta} \\
& =1_{\beta} 1_{\alpha} a+0_{\beta}=1_{\alpha \beta} a+0_{\beta}=a \psi_{\alpha, \alpha \beta} \phi_{\alpha \beta, \beta}
\end{aligned}
$$

which verifies (R4).

For any $a \in R_{\alpha}$ and $b \in R_{\beta}$, we get

$$
a b=1_{\alpha \beta}(a b)=\left[\left(1_{\alpha \beta} a\right) 1_{\alpha \beta}\right] b=\left(1_{\alpha \beta} a\right)\left(1_{\alpha \beta} b\right)=a \psi_{\alpha, \alpha \beta} \cdot b \psi_{\beta, \alpha \beta}
$$

as required.

The next example shows that not all semirings which are subdirect products of a ring and a lattice can be obtained by our construction. The example following it illustrates the fact that our construction does not generally produce the direct product. Thus the construction discussed in this section represents a strictly intermediate case between general subdirect products and the direct product of a ring and a lattice.

Example 2. Let $R$ denote the Boolean ring of all subsets of $\mathbb{N}$. Pick any ideal of $R$ which is not a retract, for instance, the ideal $I$ of all finite sets. Then $S$ $=I \times\{0\} \cup R \times\{1\}$ is a subdirect product of $R$ and the lattice $2=\{0,1\}$ (see Figure 4). However, $S$ cannot be obtained by our construction since there is no map $\psi_{1,0}$ with the required properties. 


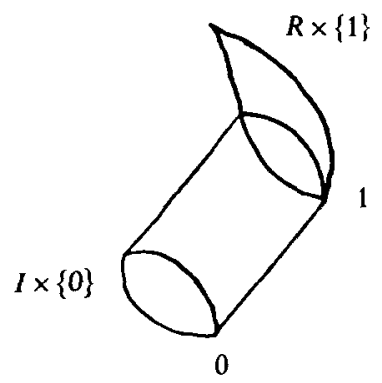

Figure 4

Example 3. Let $A$ and $B$ be the Boolean rings of all subsets of $\mathbb{N}$ and of $\mathbb{N}_{0}$ $=\mathbb{N} \cup\{0\}$, respectively. Then $A \cong B$ and $S=A \times\{0\} \cup B \times\{1\}$ is a lattice of rings. Since

$$
(\mathbb{N}, 0)+(\varnothing, 1)=(\mathbb{N}, 1) \neq\left(\mathbb{N}_{0}, 1\right)
$$

$S$ is directly indecomposable (see Figure 5).

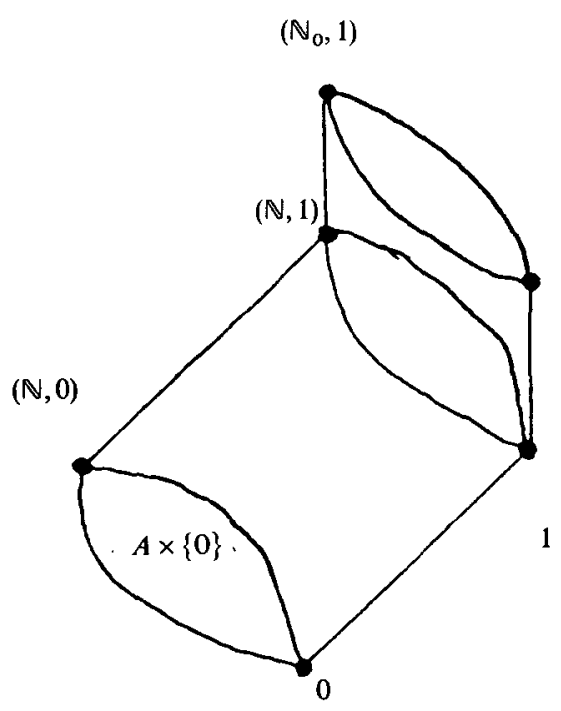

Figure 5

This seems to be in disagreement with the second assertion of ([6], Theorem 6).

\section{REFERENCES}

1. E. Graczyńska, On the sums of double systems of some universal algebras I, II, Bull. Acad. Polon. Sci. Sèr. Sci. Math. Astronom. Phys. 23 (1975), 509-513, 1055-1058.

2. D. B. McAuster, Partially ordered inverse semigroups, Conference on Semigroups (Tulane, 1978). 
3. G. Michler und R. WILle, Die primitiven Klassen arithmetischer Ringe, Math. Z. 113 (1970), 369-372.

4. M. H. A. Newman, A characterisation of Boolean lattices and rings, J. London Math. Soc. 16 (1941), 256-272.

5. M. Petrich, Introduction to Semigroups (Merrill, Columbus, 1973).

6. G. Rodrlouez, Una classe di semianelli unione di anelli con unità, Atti Sem. Mat. Fis. Univ. Modena 23 (1974), 1-21.

7. H. Werner und R. WILLE, Charakterisierungen der primitiven Klassen arithmetischer Ringe, Math. Z. 115 (1970), 197-200.

8. V. N. SALII, K teorii inversnych polukolec, Izv. Vyšs. Uč. Zav Mat. 3 (1969), 52-60.

U:MVi:RSITÄT OldenBurg

D-2900 OldenburG

F.R. Germany 